\title{
Contribution of NMDA and AMPA Receptors to Temporal Patterning of Auditory Responses in the Inferior Colliculus
}

\author{
Jason Tait Sanchez, ${ }^{1,2}$ Donald Gans, ${ }^{1,2}$ and Jeffrey J. Wenstrup ${ }^{1}$ \\ ${ }^{1}$ Department of Neurobiology, Northeastern Ohio Universities College of Medicine, Rootstown, Ohio 44272, and ${ }^{2}$ School of Speech Pathology and \\ Audiology, Kent State University, Kent, Ohio 44270
}

\begin{abstract}
Although NMDA receptors (NMDARs) are associated with synaptic plasticity, they form an essential part of responses to sensory stimuli. We compared contributions of glutamatergic NMDARs and AMPA receptors (AMPARs) to auditory responses in the inferior colliculus (IC) of awake, adult mustached bats. We examined the magnitude and temporal pattern of responses to tonal signals in single units before, during, and after local micro-iontophoretic application of selective antagonists to AMPARs [NBQX (1,2,3,4-tetrahydro-6-nitro2,3-dioxo-benzo[f]quinoxaline-7-sulfonamide)] and NMDARs [CPP (( \pm$) 3$-(2-carboxypiperazin-4-yl)-propyl-1-phosphonic acid)]. Combined blockade of AMPARs and NMDARs eliminated excitatory responses in nearly all neurons, whereas separate blockade of each receptor was quantitatively similar, causing substantial $(>50 \%)$ spike reductions in $\sim 75 \%$ of units. The major result was that effects of receptor blockade were most closely related to the first-spike latency of a unit. Thus, AMPAR blockade substantially reduced spikes in all short-latency units $(<12 \mathrm{~ms}$ ) but never in long-latency units $(\geq 12 \mathrm{~ms})$. NMDAR blockade had variable effects on short-latency units but reduced spikes substantially for all long-latency units. There were no distinct contributions of AMPARs and NMDARs to early and late elements of responses. Thus, AMPAR blockade reduced early (onset) spikes somewhat more effectively than NMDAR blockade in short-latency units, but NMDAR blockade reduced onset spikes more effectively in long-latency units. AMPAR and NMDAR blockade were equally effective in reducing later elements of sustained responses in short-latency units, whereas NMDAR blockade was much more effective in long-latency units. These results indicate that NMDARs play multiple roles for signal processing in adult IC neurons.
\end{abstract}

Key words: CPP; NBQX; glutamate; temporal pattern; latency; mustached bat

\section{Introduction}

As the principal excitatory neurotransmitter in the CNS, glutamate binds to postsynaptic receptors that directly gate cation channels. AMPA receptors (AMPARs) mediate rapid-onset, depolarizing postsynaptic currents, whereas NMDA receptors (NMDARs) mediate depolarizing currents with more gradual onset and longer duration. NMDAR-mediated currents often require AMPAR-mediated depolarization to remove extracellular $\mathrm{Mg}^{2+}$ blockade of NMDAR-associated channels (Ozawa et al., 1998). The later excitatory response mediated by NMDARs contributes to synaptic plasticity by allowing $\mathrm{Ca}^{2+}$ entry that activates signal transduction cascades. In some neurons (e.g., in sensory systems), NMDARs may contribute essentially to the pattern of action potentials that encodes information (Sillito et al., 1990; Feldman and Knudsen, 1994). Here, NMDARs play roles related

Received July 7, 2006; revised Jan. 17, 2007; accepted Jan. 18, 2007.

This work was supported by Research Grant R01 DC00937 (J.J.W.) and National Research Service Award F31 DC007298 (J.T.S.) from the National Institute on Deafness and Other Communication Disorders of the United States Public Health Service. We thank Z. M. Fuzessery, A. Galazyuk, Y. Lu, and B. Schofield for discussion of the data and manuscript. We are grateful to the Wildlife Section of the Ministry of Agriculture, Land, and Marine Resources of Trinidad and Tobago, West Indies, for permission to exports bats. Work by J.T.S. was in partial fulfillment of a PhD in audiology at Kent State University.

Correspondence should be addressed to Jeffrey J. Wenstrup, Department of Neurobiology, Northeastern Ohio Universities College of Medicine, 4209 State Route 44, P.0. Box 95, Rootstown, OH 44272. E-mail: jjw@neoucom.edu.

DOI:10.1523/JNEUROSCI.2894-06.2007

Copyright $\odot 2007$ Society for Neuroscience $\quad$ 0270-6474/07/271954-10\$15.00/0 to temporal integration and timing of neural discharges: AMPARs mediate precisely timed initial spikes, whereas NMDARs mediate later spikes in response to sensory input (Binns, 1999; Kelly and Zhang, 2002; Blitz and Regehr, 2003).

This study examines how AMPARs and NMDARs contribute to the timing of neural discharges in the auditory system, where the time of occurrence (latency) of action potentials after a sound and their temporal pattern are significant. In the inferior colliculus (IC), studied here, in vitro and in vivo studies generally reinforce the view that AMPARs and NMDARs mediate early and late elements, respectively, of temporal response patterns (Faingold et al., 1989; Feldman and Knudsen, 1994; Zhang and Kelly, 2001; Ma et al., 2002; Wu et al., 2004). However, in vivo blockade of AMPARs in IC does not always eliminate early spikes as expected (Feldman and Knudsen, 1994; Zhang and Kelly, 2001), and in vitro studies of other systems reveal fast postsynaptic responses mediated by NMDARs (D’Angelo et al., 1990; Wolszon et al., 1997). A goal of this study is to clarify contributions of AMPARs and NMDARs to temporal discharge patterns in a functioning, adult neural system.

A second goal is to examine whether the slower onset of NMDAR-mediated postsynaptic currents contributes to longer first-spike latencies. Response latency is significant because it affects integrative responses to interaural time delays and to temporally distributed elements in complex sounds. It may also influence the timing of behavioral responses (Casseday and Covey, 
1996). In IC, response latencies vary from $<5$ to $>30 \mathrm{~ms}$ (Langner et al., 1987; Parks and Pollak, 1993; Le Beau et al., 1996). Because latencies of ascending excitatory inputs to IC rarely exceed 10 ms (Haplea et al., 1994; Klug et al., 2000), synaptic mechanisms likely contribute to delayed responses of IC neurons. Several studies suggest that inhibition cannot account for this range of latencies (Le Beau et al., 1996; Lu et al., 1997; Fuzessery et al., 2003).

In adult animals, we examined roles of AMPARs and NMDARs through extracellular recording of single IC neurons in conjunction with local iontophoretic application of glutamate receptor blockers. We found that NMDARs mediate fast or onset responses as well as slow or sustained responses, often appearing to act independently of AMPARs. Furthermore, our data suggest that long-latency excitatory responses to tones are mediated primarily by NMDARs.

\section{Materials and Methods}

Single-unit auditory responses were recorded from the IC of awake mustached bats (Pteronotus parnellii) captured from Trinidad and Tobago, West Indies. All procedures used in this study are in accordance with the guidelines set by the Society for Neuroscience and were approved by the Institutional Animal Care and Use Committees at the Northeastern Ohio Universities College of Medicine and Kent State University.

Surgical procedures. Eight bats provided data used in this study. Based on size and time of capture relative to the reproductive cycle of Trinidadian mustached bats, all were judged to be adults $>1$ year of age. Before surgery, an animal received intraperitoneal injections of butorphanol (5 $\mathrm{mg} / \mathrm{kg}$, Torbugesic; Fort Dodge Animal Health, Fort Dodge, IA), atropine $(0.06 \mathrm{mg} / \mathrm{kg}$, Atropine SA; Phoenix Scientific, St. Joseph, MO), dexamethasone sodium phosphate $(2 \mathrm{mg} / \mathrm{kg}$; American Pharmaceutical Partners, Los Angeles, CA), and amoxicillin (11 mg/kg). The animal was anesthetized with isoflurane inhalation (1.5-2.0\% in oxygen; Abbott Laboratories, North Chicago, IL).

After the anesthetic abolished nociceptive reflexes, the hair on the dorsal surface of the animal's head was removed using a depilatory lotion. A midline incision was made in the skin, and the underlying muscles were reflected laterally to expose the skull. The skull surface was cleaned, and a tungsten ground electrode was cemented into the skull over the cerebrum. A metal pin was also cemented onto the skull to secure the head to a stereotaxic apparatus used during physiological experiments. An opening $(\sim 0.5 \mathrm{~mm})$ was then made in the skull overlying the dorsal surface of the IC. After surgery, a local anesthetic (Lidocaine) was applied to the surgical area, and the animal was returned to the holding cage. A $2 \mathrm{~d}$ recovery period was allotted before starting physiological experiments.

Acoustic stimulation and data acquisition. Acoustic stimulation was computer controlled and included noise and tone bursts $(0.5 \mathrm{~ms}$ rise-fall time, four per second). Noise bursts (11 ms duration) were used as search stimuli, and tone bursts (4-61 ms duration) were used in all qualitative and quantitative tests. Acoustic stimuli were digitally synthesized and downloaded onto a digital signal processing card (AP2 Multi-Processor DSP card; Tucker-Davis Technologies, Alachua, FL), converted to analog signals at a sampling rate of $500 \mathrm{kHz}$ (model DA3-2; Tucker-Davis Technologies), filtered (model FT6-2; Tucker-Davis Technologies), attenuated (model PA4; Tucker-Davis Technologies), summed (model SM3; Tucker-Davis Technologies), amplified (model HCA-800II; Parasound, San Francisco, CA), and sent to a loudspeaker (Infinity EMIT-B; Harmon International Industries, Woodbury, NY). The loudspeaker was placed $10 \mathrm{~cm}$ in front of the animal and $25^{\circ}$ into the sound field contralateral to the IC under study.

The output of the acoustic system was calibrated over a frequency range of $10-120 \mathrm{kHz}$ using a condenser microphone (model 4135; Brüel and Kjaer, Nærum, Denmark) placed in a position normally occupied by the animal's head. The system response has a gradual roll-off of $\sim 3 \mathrm{~dB}$ per $10 \mathrm{kHz}$. Harmonic distortion was not detectable $60 \mathrm{~dB}$ below the
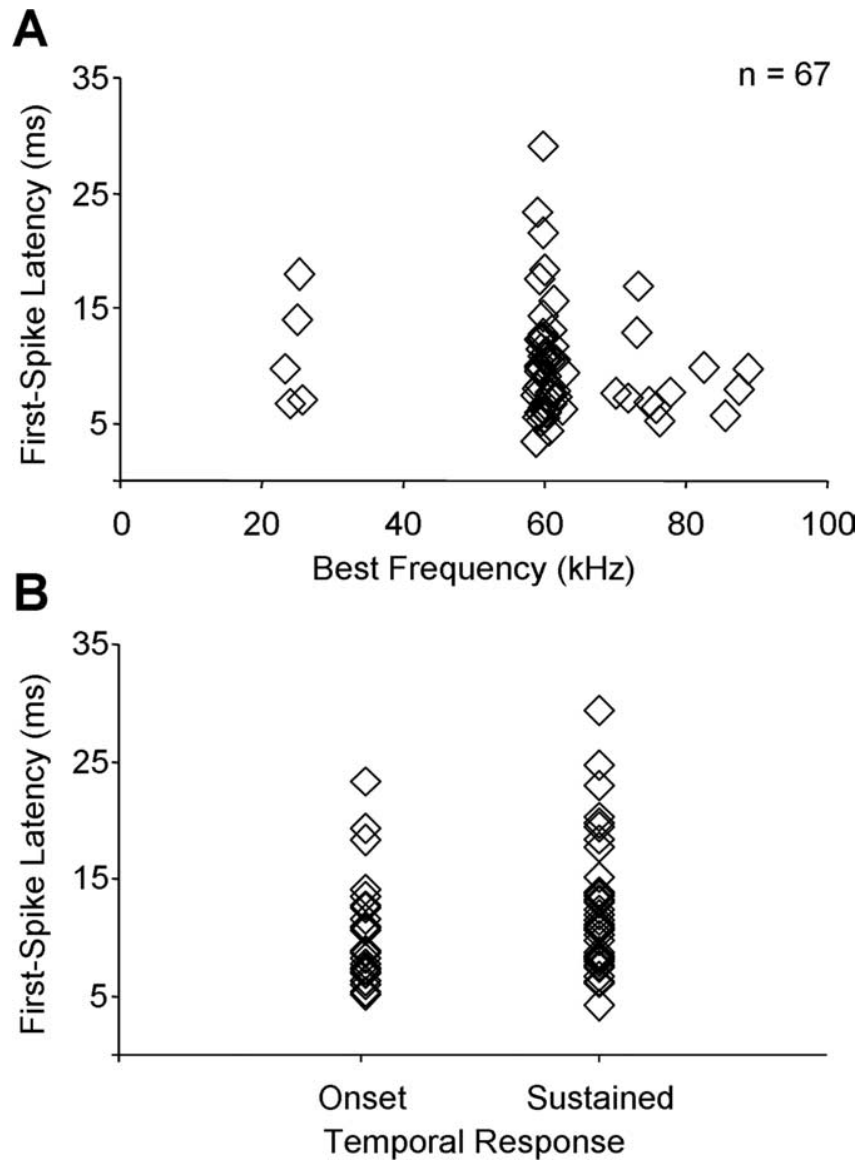

Figure 1. Frequency tuning $(\boldsymbol{A})$ and temporal features $(\boldsymbol{B})$ of recorded IC units.

signal level using a fast Fourier analysis of the digitized microphone signal (model AD2; Tucker-Davis Technologies).

Recording procedure. Recordings and micro-iontophoresis were similar to those described previously (Nataraj and Wenstrup, 2005, 2006). Briefly, physiological experiments were conducted inside a single-walled Industrial Acoustics (Winchester, UK) chamber lined with polyurethane foam to reduce echoes. On experimental days, the animal was placed in a stereotaxic apparatus inside the heated acoustic chamber. If at any time the animal showed signs of discomfort or distress, it was lightly sedated with butorphanol $(0.05 \mathrm{mg} / \mathrm{kg}$, s.c. $)$ or removed from the stereotaxic apparatus. The animal was offered water from a medicine dropper between electrode penetrations. The recording sessions did not exceed $6 \mathrm{~h}$ and were limited to one recording session per day.

All data were obtained from well isolated single units, characterized by stable amplitude, consistent shape, and a signal-to-noise ratio exceeding 5:1. Physiological recordings were amplified, filtered $(600-6000 \mathrm{~Hz})$, and sent through a spike-signal enhancer (model 40-46-1; Fredrick Haer and Company, Bowdoin, ME) before being digitized at a sampling rate of $40 \mathrm{kHz}$ (model AD2; Tucker-Davis Technologies). The digitized signal was uploaded to the computer via a second AP2 digital signal processor (Tucker-Davis Technologies). The custom-made software calculated the time of occurrence of spikes and displayed poststimulus time histograms (PSTHs), raster plots, and basic statistics of the neural responses in real time. The output of the spike preconditioner and spike discriminator was displayed on a computer monitor.

Once a single unit was isolated, its best frequency (BF) and minimum threshold (MT) were determined. BF is defined as the frequency requiring the lowest sound pressure level to elicit consistent, stimulus-locked action potentials, and MT is defined as the lowest sound level at the BF to elicit consistent, stimulus-locked action potentials. The term "best frequency," common in the neuroethological literature, is equivalent to "characteristic frequency" used elsewhere. Rate-level functions were ob- 
tained by systematically increasing the sound pressure level of $\mathrm{BF}$ tone bursts in $5 \mathrm{~dB}$ increments.

Single-unit recordings were obtained using a micropipette electrode mounted on a fivebarreled pipette (Havey and Caspary, 1980) for iontophoretic application of drugs. The tip of the multibarreled pipette was broken to a diameter of $15-30 \mu \mathrm{m}$; the unbroken tip of the single electrode extended 10-25 $\mu \mathrm{m}$ beyond the multibarreled pipette and was filled with 1 $\mathrm{M} \mathrm{NaCl}$ (resistance, 5-30 M $\Omega$ ). The center barrel of the multibarreled pipette was filled with 1 $\mathrm{M} \mathrm{NaCl}$ and connected to balance all currents used to apply or retain drugs. Other barrels were filled with the AMPA/kainate receptor antagonist 1,2,3,4-tetrahydro-6-nitro-2,3-dioxobenzo[f]quinoxaline-7-sulfonamide (NBQX) (5 mm, pH 9.0; Sigma, St. Louis, MO) and the NMDAR antagonist (士)3-(2-carboxypiperazin-4-yl)-propyl-1-phosphonic acid (CPP) (10 mm, pH 8.0; Sigma). NBQX is a highly selective competitive antagonist of both AMPA and kainate receptors, with no significant effect on NMDAR-mediated current (Randle et al., 1992). Although we will relate NBQX to AMPAR blockade, we cannot exclude the possibility that kainate receptors contribute to some NBQX-sensitive responses. Differential roles of AMPARs and kainate receptors are not understood in IC. To block NMDARs, CPP is both highly selective and more potent than alternatives such as AP-5 (Lodge et al., 1988). A previous in vivo study of glutamate receptor function in IC used these same receptor antagonists (Zhang and Kelly, 2001).

On the day of the experiment, each drug was dissolved in $0.9 \% \mathrm{NaCl}$ vehicle. Each barrel of the multibarreled pipette was connected via a silver wire to a separate channel of a microiontophoresis current generator (programma-

ble current generator, model 6400; Dagan, Minneapolis, MN). NBQX and $\mathrm{CPP}$ were retained using positive currents $(+10 \mathrm{nA})$ and ejected using negative currents ( -20 to $-80 \mathrm{nA})$. In initial studies, we obtained dose-response curves to determine the appropriate current settings for application of these drugs. In most cases, application currents greater than $-30 \mathrm{nA}$ caused no further reduction in spikes than $-30 \mathrm{nA}$. To ensure maximum blockade of receptors, we typically used $-40 \mathrm{nA}$ application current. In some cases, higher currents were used when drug application with $-40 \mathrm{nA}$ current failed to show an effect. We also conducted current/pH controls using drug vehicle adjusted in $\mathrm{pH}$ to correspond to the NBQX or CPP solutions. We observed no effect on unit discharges of current injection as high as $100 \mathrm{nA}$ through these $\mathrm{pH}$ adjusted vehicle solutions.

The piggyback multibarreled assembly was advanced into the brain by a hydraulic micropositioner (model 650; David Kopf Instruments, Tujunga, CA). Drug injection times and ejection currents varied depending on the effect of the drug(s) monitored both audiovisually and quantitatively. With currents used in this study, effects of NBQX and CPP could be observed as early as $2 \mathrm{~min}$ after the start of drug application, whereas steady-state responses were usually observed after 4-8 min. Complete or partial recovery could be observed as early as $4 \mathrm{~min}$ or as long as $1 \mathrm{~h}$ after drug application was stopped and depended on the drug, ejection currents, and the duration of drug application. In cases in which AMPAR blockade by NBQX had no effect or only a small effect on discharge rate, we verified that the drug was ejected and effective at other recording sites in the same penetration. We only included NBQX-insensitive units for which NBQX application was verified in this way.

Data analysis. Quantitative data were obtained for all units using 32
B
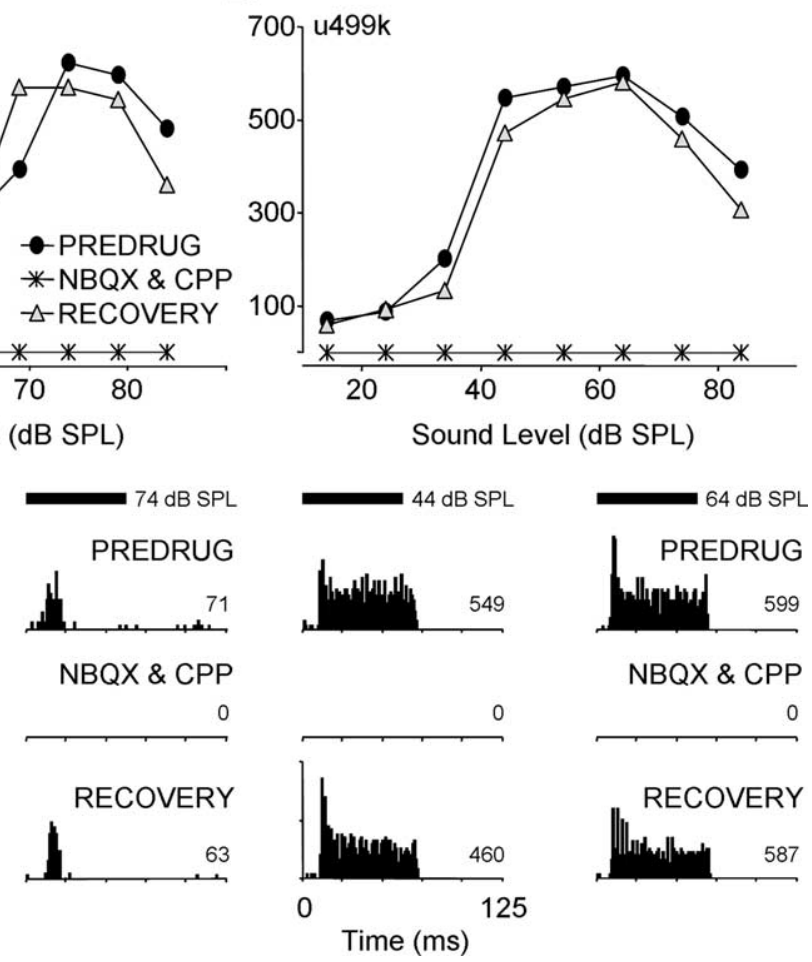

Time $(\mathrm{ms})$

Figure 2. Combined blockade of AMPARs and NMDARs eliminates excitation in IC single units. Rate-level functions and PSTHs ( ( Combined application of NBQX and CPP completely eliminated unit responses at each sound level tested. The bin width and currents are as follows: in $A$, NBQX \& CPP, $18 \mathrm{~min},-40 \mathrm{nA}$ each; RECOVERY, $4 \mathrm{~min}$; in $B$, NBQX \& CPP, $15 \mathrm{~min},-40 \mathrm{nA}$ each; RECOVERY, 9 min. SPL, Sound pressure level.

repetitions of each stimulus. Temporal discharge patterns were categorized as onset or sustained. Onset responses exhibited one or a few well timed action potentials per stimulus, followed by few or no spikes $(<20 \%$ of total spikes) for the remainder of the stimulus duration. Sustained units discharged action potentials throughout the duration of the stimulus. This category included responses characterized by a brief silent period after the initial burst of action potentials. To express the timing of the first spike of the response of a unit to a particular stimulus, we calculated the median value across 32 stimulus presentations and subtracted the acoustic travel time of 0.3 ms ("median first-spike latency"). When comparing median first-spike latencies across recorded units, we report the minimum value of the median first-spike latency obtained across the sound levels tested in a rate-level function ("minimum first-spike latency"). Statistical analyses were performed with an error level $(\alpha)$ of 0.05 . Mean values are reported with the corresponding SE. Wherever possible, details of statistical tests are reported in related figure legends.

\section{Results}

We examined effects of glutamatergic receptor antagonists on 67 single units from the IC of adult mustached bats. BFs ranged from 22.0 to $87.4 \mathrm{kHz}$, with most tuned near or above $58 \mathrm{kHz}$. In recordings before drug application, 32 units had sustained discharge patterns and 35 units had onset discharge patterns. Minimum first-spike latency for these units ranged from 4.3 to 29.4 $\mathrm{ms}$, with an average response latency of $9.5 \pm 0.6 \mathrm{~ms}$. Figure $1, A$ and $B$, shows the distributions of response latency as functions of $\mathrm{BF}$ and temporal response pattern, respectively. 
General effects of glutamate receptor antagonists

Together, ionotropic AMPA and NMDA glutamate receptor antagonists blocked nearly all action potentials evoked by tone bursts. Figure 2 shows rate-level functions and PSTHs for two IC units obtained before, during, and after simultaneous applications of NBQX to block AMPARs and CPP to block NMDARs. For both the onset unit in Figure $2 A$ and the sustained unit in Figure $2 B$, combined application of NBQX and CPP completely blocked both spontaneous action potentials and auditory evoked activity at each sound level tested. In 16 of 18 units tested with combined drug application, spike reduction was $>90 \%$. This sample included both onset and sustained response patterns with a range of first-spike latencies. These results indicate that excitatory responses in the mustached bat's IC are mediated primarily by ionotropic glutamate receptors. Furthermore, they suggest that the drug application parameters used here (concentration and application current) were sufficient to block nearly all glutamate-based excitatory responses.

Separate application of NBQX and CPP reduced spikes substantially in most units across the suprathreshold sound levels tested. Our criterion for a substantial drug effect was a 50\% reduction in response magnitude. The average spike reduction by NBQX across all tested units was $63 \%(n=59)$, whereas the average spike reduction by CPP was $67 \%(n=58)$. Separate application of NBQX caused a substantial reduction in spike discharge in $78 \%$ of tested units, whereas application of CPP alone caused a substantial reduction in discharge rate in $74 \%$ of tested units. There were no significant differences in these effects of NBQX and CPP, based on a two-sample $t$ test assuming unequal variances (for average spike reduction) and a $2 \times 2 \chi^{2}$ analysis (for numbers of units showing substantial spike reduction). In these general terms, therefore, both AMPARs and NMDARs contribute substantially and similarly to the excitatory response of IC units.

\section{Differential effects of glutamate receptor antagonists on temporal response features}

Do AMPARs and NMDARs play differential roles in the responses of IC neurons to sounds? Previous work (Zhang and Kelly, 2001; Kelly and Zhang, 2002) concluded that AMPARs contribute primarily to spikes in onset patterns and in the earlier component of sustained temporal patterns, whereas NMDARs contribute primarily to spikes in the later component of sustained responses. The role of AMPARs in later spikes of sustained responses was unclear. The major finding in our study is that response latency, rather than temporal response pattern, was the major feature of IC responses related to differential roles of these receptors.

To examine whether AMPARs and NMDARs contribute differentially to response latency, we plotted the percentage of reduction in spike count as a function of the first-spike latency of a unit. Figure $3 A$ shows the effect of NBQX on both onset and sustained responses. For units with first-spike latencies $<12 \mathrm{~ms}$, NBQX application always had a substantial effect $(>50 \%$ reduction), and usually had a very strong effect ( $>90 \%$ reduction). For units with latencies $>12 \mathrm{~ms}$, however, NBQX never caused a substantial reduction in discharge rate. The effect of CPP was related differently to first-spike latency (Fig. $3 B$ ). For units with short first-spike latencies, the effect of CPP ranged from very weak to very strong. For units with increasing first-spike latency, the effect of CPP was stronger; it always caused a substantial reduction in spikes for units with latencies of $\geq 12 \mathrm{~ms}$. These latency-dependent effects of NBQX and CPP were observed
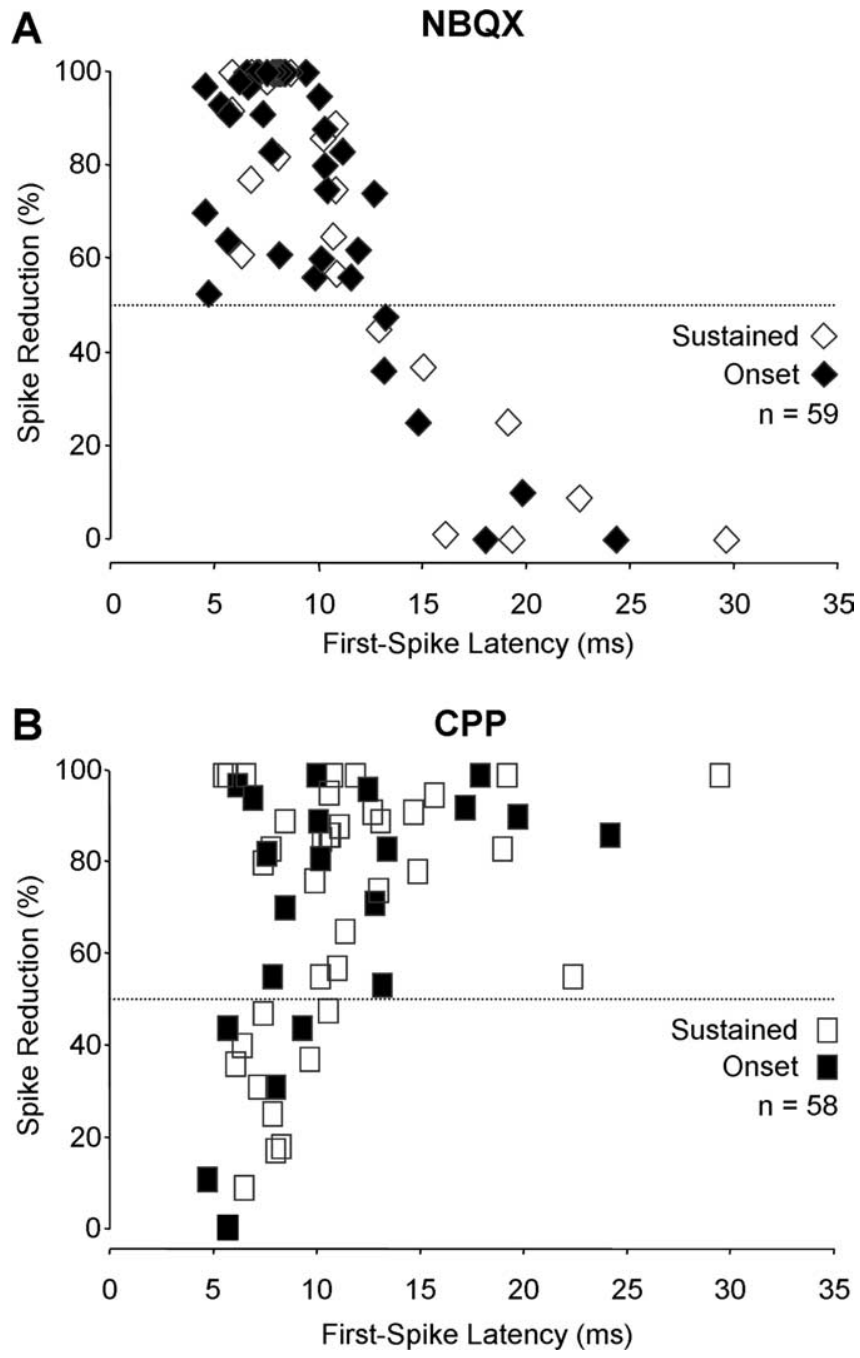

Figure 3. Glutamate receptor contributions varied with first-spike latency. Each data point shows the percentage of reduction in spikes of one unit resulting from drug application as a function of the first-spike latency of the unit. $A$, NBQX reduced spikes substantially for all units with latencies $<12$ ms but never for units with longer latencies. $B$, CPP always reduced spikes substantially for units with latencies $\geq 12$ ms but had variable effects on units with shorter first-spike latencies. The horizontal dashed line indicates the criterion for substantial drug effect based on $50 \%$ spike reduction.

across both onset and sustained units (Fig. 3). The sections below explore the relationship between latency and each temporal response pattern in greater detail.

Roles of AMPARs and NMDARs in onset responses Consistent with previous results in IC and elsewhere, we observed in some onset units that NBQX eliminated or substantially reduced spikes whereas CPP had little effect (Fig. 4A). However, we also recorded onset units for which CPP eliminated spikes whereas NBQX caused no reduction (Fig. $4 B$ ). Across the entire sample of onset units, both NBQX and CPP caused major spike reductions (Fig. 5A, All Onset Units). Moreover, both drugs caused a substantial reduction in spikes in most units (Fig. $5 B$, All Onset Units).

When analyzed in the context of first-spike latency, it is apparent that AMPARs play a dominant role only in short-latency onset responses (Fig. $4 A$ ). In such responses, NBQX caused an average reduction in spikes of $80 \%$ (Fig. $5 A$ ), reducing spikes substantially in all units (Fig. $5 B$ ). CPP had a lesser, but nonethe- 
less substantial, effect on short-latency onset units, causing a mean spike reduction of $50 \%$ (Fig. $5 A$ ) and substantially reducing spikes in $66 \%$ of units (Fig. $5 B$ ). For long-latency units (Fig. $4 B$ ), the roles were reversed. CPP caused a substantial spike reduction in all units (Fig. $5 B$ ), averaging $80 \%$ reduction (Fig. $5 A$ ). NBQX never caused a substantial spike reduction in long-latency units, with an average reduction of 30\% (Fig. 5). For both drugs, spike reduction was significantly different between short- and long-latency onset units. These results indicate a restricted role for AMPARs, mediating onset responses only in short-latency responses. NMDARs mediate onset spikes in both short- and longlatency units, but their role is predominant in long-latency units.

\section{Roles of AMPARs and NMDARs in sustained responses}

Across all sustained units, both NBQX and CPP caused quantitatively similar, substantial reductions in spikes. Neither the average percentage of reduction (Fig. $6 \mathrm{~A}$, All Sustained Units) nor the percentage of units showing a substantial reduction in discharge (Fig. 6B, All Sustained Units) was significantly different for CPP and NBQX application.

When sustained units were separated into short- and long-latency groups, however, there were large differences in drug effects that closely paralleled effects observed in onset units. For short-latency sustained units, NBQX caused an average reduction in spikes of $80 \%$ (Fig. $6 \mathrm{~A}$ ), reducing spikes substantially in all units (Fig. 6B). CPP had a lesser effect on short-latency units, causing a mean spike reduction of $50 \%$ (Fig. 6A) and substantially reducing spikes in $61 \%$ of units (Fig. $6 \mathrm{~B}$ ). Among long-latency units, the situation was dramatically reversed. CPP caused a substantial spike reduction in all units, averaging $80 \%$ reduction. NBQX never caused a substantial spike reduction in long-latency units, with an average reduction of $25 \%$. Differences in effects of the two drugs within each latency group were significant, and differences in effects of each drug on the two latency groups were significant (Fig. 6). These results indicate that AMPARs play a major role in mediating spikes for units with short-latency responses but only a small role in spikes of long-latency units. The results also indicate that NMDARs play a significant role in mediating spikes of the majority of short-latency units and play the dominant role in spiking of long-latency units.

\section{Roles of AMPARs and NMDARs in}

earlier and later components of sustained responses

Our results, indicating that glutamate receptor effects are related to first-spike latency, are in conflict with previous work in auditory and other systems suggesting that AMPARs and NMDARs contribute differentially to spikes in the earlier and later components of sustained responses. Here, we examine whether differential effects of receptor blockade occur among individual units
B
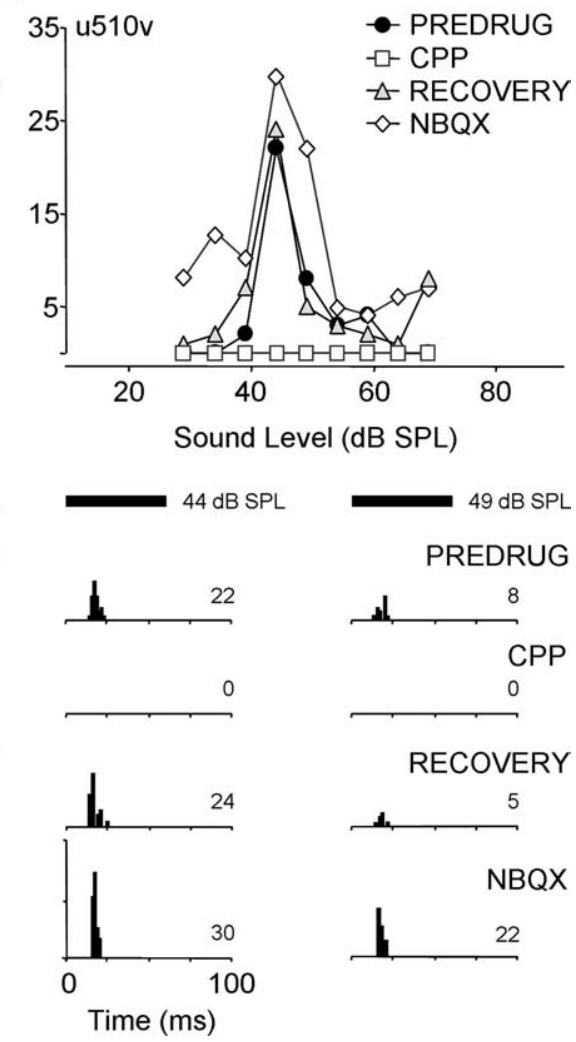

PREDRUG

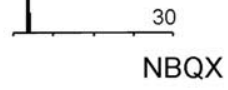

1

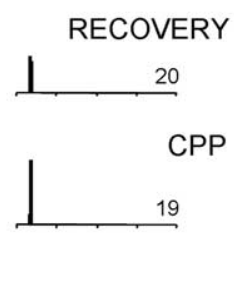

Figure 4. Blockade of AMPARs and NMDARs in onset units has different effects related to first-spike latency. Rate-level funcfollows: in $\boldsymbol{A}$, NBQX, $12 \mathrm{~min},-40 \mathrm{nA}$; RECOVERY, $7 \mathrm{~min}$; CPP, $13 \mathrm{~min},-40 \mathrm{nA}$; in B, CPP, 8 min., $-40 \mathrm{nA}$; RECOVERY, 4 min; NBQX $24 \mathrm{~min},-40 \mathrm{nA}$. See Figure 2 for protocol. SPL, Sound pressure level.

and relate them to first-spike latency across the sample of sustained units.

In some units, we observed that AMPARs mediate early spikes, whereas NMDARs mediate late spikes. For the sustained unit in Figure 7A, NBQX blocked all onset spikes (those within the initial $10 \mathrm{~ms}$ of the response) but had a reduced effect (usually $<50 \%$ ) on subsequent spikes in the response (Fig. 7A, 10-150 ms graph). For the unit in Figure $7 B, \mathrm{CPP}$ had a stronger influence on later spikes (Fig. 7B, 10-150 ms graph), eliminating all spikes in the final 30-35 ms of the response. However, two additional aspects of the response of this unit deserve mention. First, the AMPAR-mediated excitation that survived CPP application included many spikes over $\sim 30 \mathrm{~ms}$ duration (five to seven spikes per stimulus in the PSTHs). This indicates that AMPARs may contribute more to discharge patterns than just the initial spike. Second, CPP also reduced onset spikes (Fig. 7B, 0-9 ms graph), but spike reduction was substantial only at higher sound levels. These units show differential effects of NBQX and CPP consistent with previous work in IC (Zhang and Kelly, 2001).

In many other sustained units, we did not observe a strong differential effect of drugs on earlier and later spikes. For the unit in Figure 8A, NBQX completely eliminated both earlier and later components of the responses. In Figure $8 B$, CPP had a major effect on both earlier and later components of sustained discharge of a unit, whereas NBQX had only a negligible effect compared with its control. 

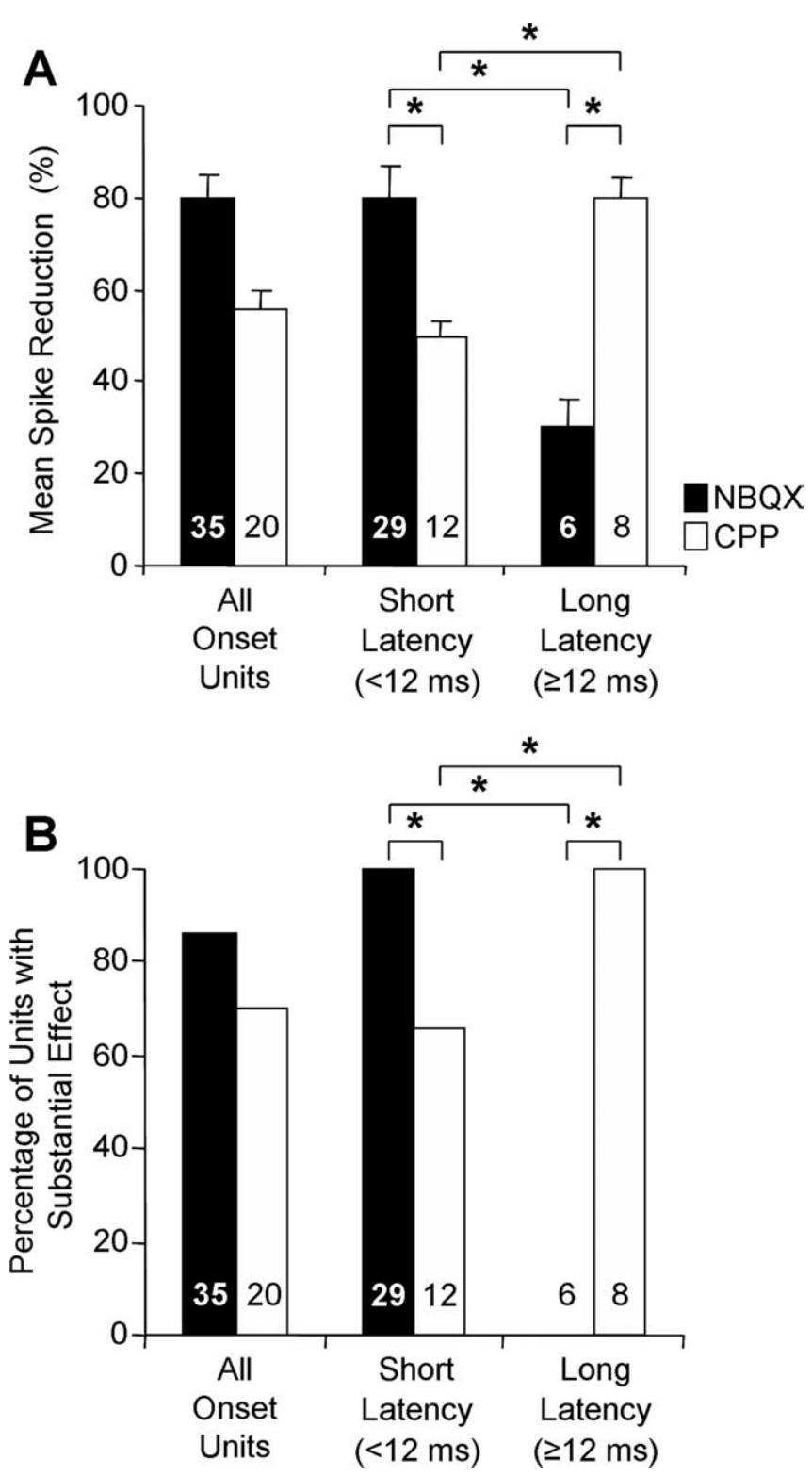

Figure 5. Contributions of AMPARs and NMDARs to onset temporal patterns depend on first-spike latency. $\boldsymbol{A}, \boldsymbol{B}$, The effects of drug application across the sample of onset units are plotted as a mean reduction in the number of spikes $(\boldsymbol{A})$ and the percentage of units showing a substantial $(>50 \%)$ reduction in spikes $(\boldsymbol{B})$. Both NBQX and CPP had major effects across the sample of onset units (All Onset Units), but this was composed of distinct subgroups based on first-spike latency. Error bars in $\boldsymbol{A}$ indicate SEM. Statistical comparisons for different drugs within a latency group and for each drug across latency groups were performed using twosample $t$ tests assuming unequal variances $(\boldsymbol{A})$ and $2 \times 2 \chi^{2}$ tests $(\mathrm{df}=1)(\boldsymbol{B}) .{ }^{*} p<0.01$. Numbers within histogram bars indicate sample size. No tests were performed on the group All Onset Units because it was composed of unequal numbers of short- and long-latency units.

To further evaluate the effects of the selective antagonists on the time course of sustained responses, we plotted the percentage of reduction in unit responses as a function of timing after the first spike, computed in $10 \mathrm{~ms}$ bins. These plots (Fig. 9) thus display the effect of drug application on different components of temporal response patterns, independent of first-spike latency. Figure 9, $A$ and $B$, reveals how variable were the effects of drug application on the temporal pattern of responses in different units. In some units, NBQX eliminated the entire response (Fig. $9 A$, unit 1), but in others, it had only minor effects across the response duration (units 3 and 4 ). The effect of CPP was similarly
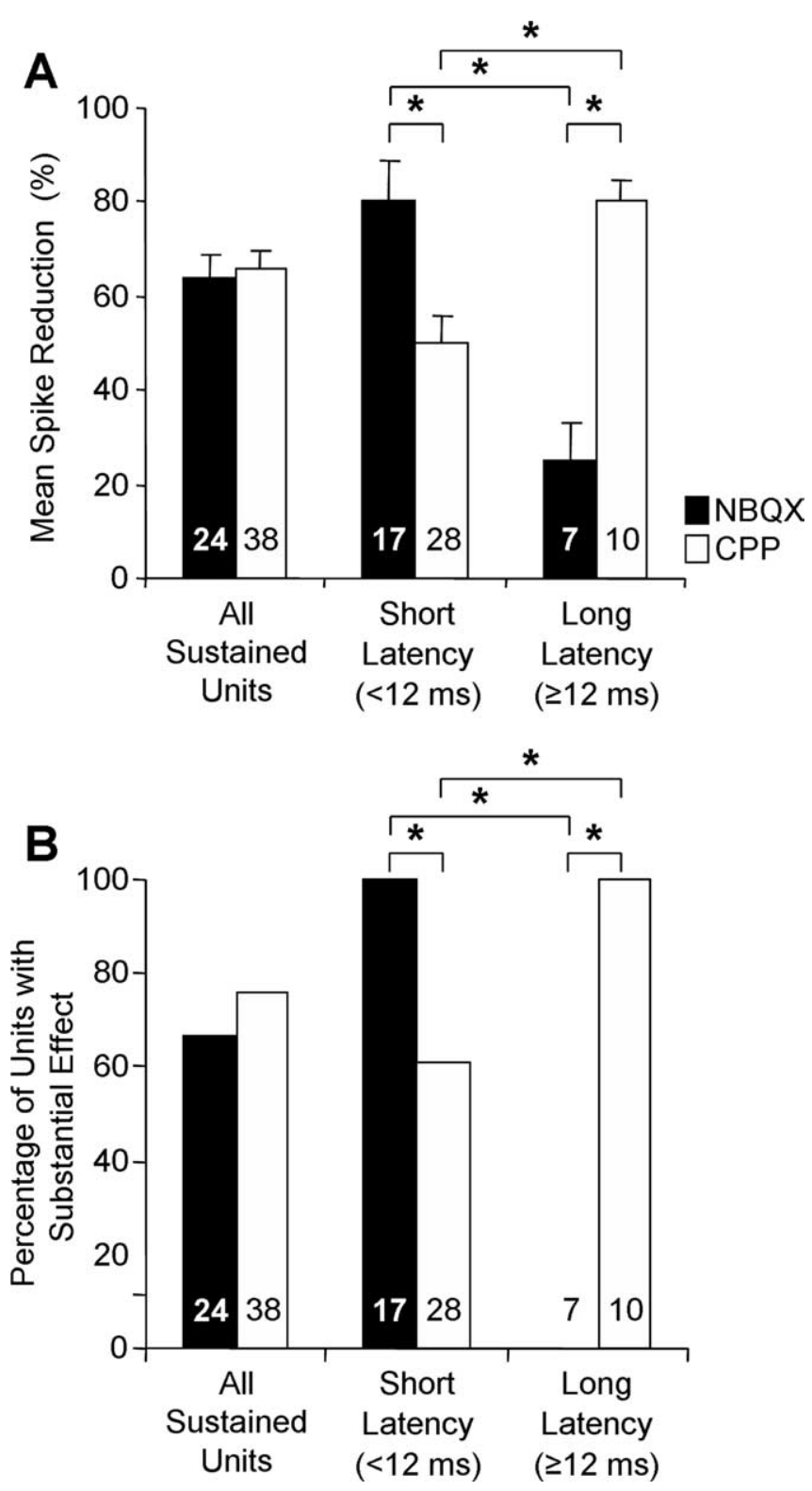

Figure 6. Contributions of AMPARs and NMDARs to responses of sustained units depend on first-spike latency. Across the entire sample of sustained units, both NBQX and CPP reduced spikes substantially (All Sustained Units). For short-latency units, both NBQX and CPP effectively reduced spikes, but NBQX had a significantly stronger effect. For long-latency units, CPP reduced spikes very effectively, whereas NBQX was ineffective in reducing spikes. See Figure 5 for protocol.

variable. In Figure 9B, two units (units 7 and 8) showed strong effects of CPP only during the later component of the response, consistent with previous studies of the time course of NMDAmediated excitation. However, even here the time course was quite different: in unit 7, CPP had a strong effect as early as 10-20 $\mathrm{ms}$ after the initial response, whereas in unit 8, the strong CPP effect began much later, 50-60 ms after the initial response. In two other units (units 5 and 6), CPP had a strong effect throughout the entire time course of the response.

To examine how these variations relate to first-spike latency, we plotted the effect of drug application on the time course of responses for the short- and long-latency groups of sustained units. Figure $9 C$ reveals several features of NBQX application. First, NBQX caused a much stronger reduction in spikes for short-latency units than for long-latency units, in agreement with 
Figure 6. Second, NBQX had similar effects throughout the response duration: in both short- and long-latency units, there was no significant difference in spike reduction for the first $10 \mathrm{~ms}$ of the response (0-9 ms bin) compared with the remainder of the response (10-69 ms). These results suggest that, in general, AMPARs contribute similarly to early and late spikes in temporal response patterns, but their role is much stronger in shortlatency units than in long-latency units. Additional analysis revealed that, for the two latency groups, the effect of NBQX on onset units was indistinguishable from its effects on either the earlier or later components of sustained units (Fig. 9E). Thus, differences related to response latency far outweigh differences in temporal response pattern in understanding the role of AMPARs in IC responses.

CPP application, on average, caused a substantial reduction in spikes throughout the time course of responses (Fig. 9D). For both short- and long-latency populations, there was a stronger, statistically significant effect on later spikes than on spikes in the initial $10 \mathrm{~ms}$ of the response. However, the effect of CPP on early spikes was nonetheless substantial in both populations. Figure $9 F$ shows that the effect of $\mathrm{CPP}$ on onset units was indistinguishable from its effects on the onset component of sustained units. These results suggest that NMDARs contribute significantly to action potentials throughout the time course of responses, even for many units with short first-spike latencies.

Finally, we compared spike reduction by NBQX and CPP in different components of the sustained responses, based on analyses shown in Figure 9, $C$ and $D$. For short-latency units, spikes in the initial $0-9$ ms bin were reduced more strongly by NBQX than by CPP ( $p<0.01$, twosample $t$ test assuming unequal variances). For later spikes (10-69 ms), there was no difference in effects of NBQX and CPP ( $p>0.2$, two-sample $t$ test assuming unequal variances). For long-latency responses, CPP had a much stronger effect than NBQX on both early and late components of responses.

\section{Discussion}

Among IC neurons, excitatory responses to simple tonal stimuli depend on ionotropic glutamate receptors. We compared effects of pharmacological blockade of AMPARs and NMDARs on the timing and magnitude of excitatory responses. The major finding was that blockade of these receptors had differential effects related to
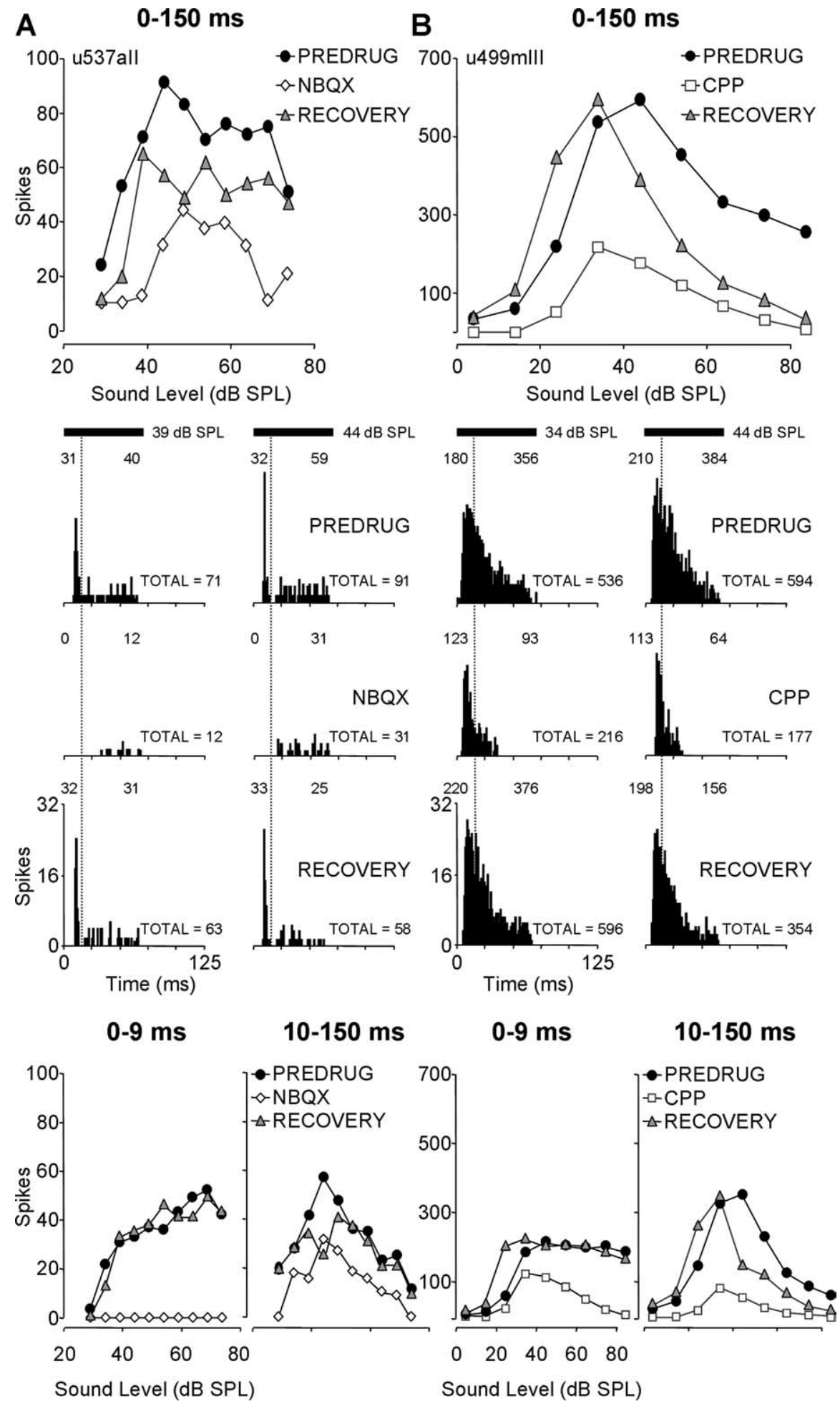

Figure 7. Blockade of glutamate receptors differentially affected early and late components of some sustained units. In two units, drug effects were analyzed for early $(0-9 \mathrm{~ms})$ and late $(10-150 \mathrm{~ms})$ response components. $A$, NBQX primarily blocked early spikes in this IC unit. BF, $57.9 \mathrm{kHz}$; MT, $39 \mathrm{~dB} \mathrm{SPL}$; minimum latency, $8.1 \mathrm{~ms}$. The rate-level function of an entire response $(0-150$ $\mathrm{ms}$; top), PSTHs (middle), and rate-level functions of early $(0-9 \mathrm{~ms})$ and late $(10-150 \mathrm{~ms})$ components (bottom) relative to the beginning of response are shown. $\boldsymbol{B}$, CPP blocked later spikes more effectively in this unit (BF, 57.9 kHz; MT, $14 \mathrm{~dB} \mathrm{SPL}$; minimum latency, $10.2 \mathrm{~ms}$ ), although (PP substantially reduced early spikes at higher sound levels. Vertical dotted lines in PSTHs separate early and late spikes. Numbers at the bottom right of each PSTH represent total spikes for a $150 \mathrm{~ms}$ time window; numbers in windowed regions above PSTHs separately display spike counts for early and late excitation. Drug application times and currents are as follows: in $A$, NBQX, $18 \mathrm{~min},-80 \mathrm{nA}$; RECOVERY, $10 \mathrm{~min}$; in $B$, CPP, $12 \mathrm{~min},-40 \mathrm{nA}$; RECOVERY, $11 \mathrm{~min}$. See Figure 2 for protocol. SPL, Sound pressure level. 
A

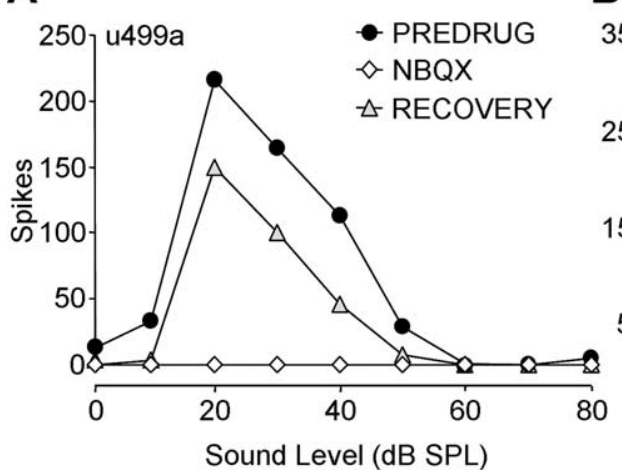

B
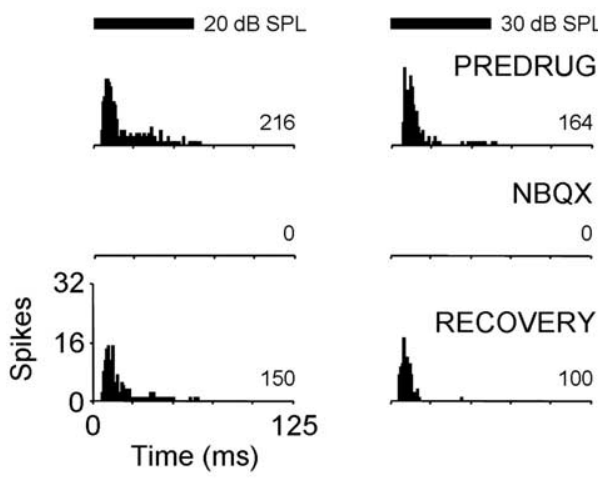

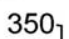

$-1$ $\triangle$ RECOVERY $250 . \approx$ NBQX 150 $\frac{{ }_{\text {Sound Level (dB SPL) }}^{40}}{40}$

$64 \mathrm{~dB}$ SPL
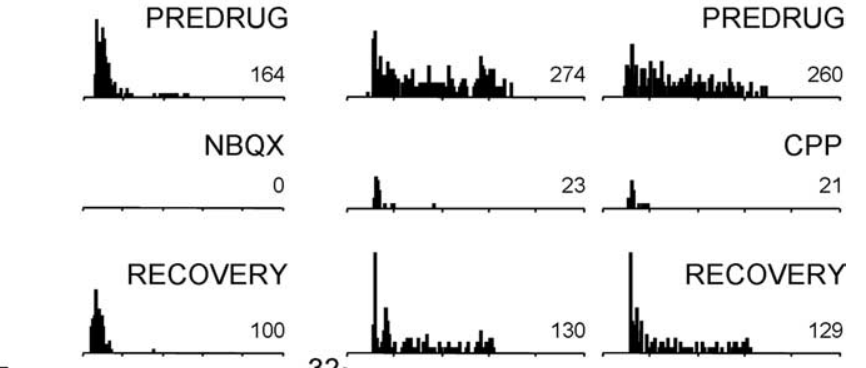

32

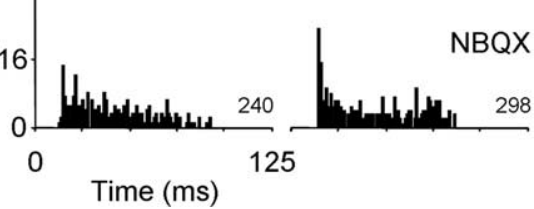

Figure 8. Blockade of glutamate receptors substantially reduced both early and late spikes in some sustained units. $A$, NBQX completely eliminated all spikes in this unit. BF, $59.5 \mathrm{kHz}$; MT, $9 \mathrm{dBSPL}$; minimum latency, $7.7 \mathrm{~ms}$. B, (PP eliminated nearly all early and late spikes in this unit, whereas $\mathrm{NBQX}$ did not reduce spikes relative to recovery. BF, $58.2 \mathrm{kHz} ; \mathrm{MT}, 39 \mathrm{dBSPL}$; minimum latency, $15.1 \mathrm{~ms}$. Drug application times and currents are as follows: in $A$, NBQX, $16 \mathrm{~min},-40 \mathrm{nA}$; RECOVERY, $8 \mathrm{~min}$; in B, CPP, $10 \mathrm{~min},-40$ $\mathrm{nA}$; RECOVERY, $18 \mathrm{~min}$; NBQX, $12 \mathrm{~min},-60 \mathrm{nA}$. See Figure 2 for protocol. SPL, Sound pressure level.

the first-spike latency of a neuron rather than to its temporal response pattern. NBQX, a selective AMPA/kainate antagonist, blocked most spikes in both early and late components of the response of a neuron, but only for neurons with first-spike latencies $<12 \mathrm{~ms}$. CPP, an antagonist to NMDARs, also blocked spikes in both early and late components of the response of a neuron. This was true even in some neurons with short first-spike latencies. In neurons with long first-spike latencies ( $\geq 12 \mathrm{~ms}$ ), CPP blocked nearly all onset and sustained spikes. These results, observed in awake, adult animals, suggest that functions of NMDARs in sensory-evoked responses are more diverse than generally understood. They also suggest a broad range of functional NMDAR classes among IC neurons.

\section{AMPAR- and NMDAR-mediated excitation in IC}

We draw several conclusions regarding NMDAR-mediated excitation in IC. First, NMDARs mediate excitatory responses of some IC neurons in the absence of AMPAR-mediated depolarization. This agrees closely with conclusions of previous in vivo iontophoretic studies in IC (Feldman and Knudsen, 1994; Zhang and Kelly, 2001). Furthermore, it supports in vitro IC studies showing that NMDARs conduct current at resting membrane potentials when AMPARs are blocked (Ma et al., 2002; Sivaramakrishnan and Oliver, 2005). Evidence for the independent action of NMDARs has also been reported previously [e.g., in the

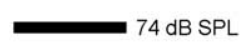
PREDRUG

CPP

spinal cord (Davies and Watkins, 1983), lateral geniculate nucleus (Sillito et al., 1990), and mouse barrel cortex (Fleidervish et al., 1998)].

A second conclusion is a major extension of the first. NMDARs almost exclusively mediate excitatory responses in neurons with long first-spike latency. This has not been reported previously in the auditory system. It is similar to results indicating that long-latency lateral geniculate responses are mediated exclusively by NMDARs (Heggelund and Hartveit, 1990), but that finding was disputed (Kwon et al., 1991). An exclusive role for NMDARs in excitation has been described in some hippocampal neurons (Kirson and Yaari, 2000). For IC neurons with long first-spike latencies, we predict that they contain NMDARs that conduct depolarizing current at resting membrane potentials, that they vary in the rise time of NMDAR-mediated currents in a manner related to their response latency, and that functional AMPARs are few or absent.

A third conclusion is that NMDARs can contribute significantly to early excitation. In some neurons, NMDAR blockade eliminated all spikes occurring as early as $6 \mathrm{~ms}$ after stimulus onset. Although we cannot know the latencies of inputs to such neurons, it is clear that their NMDAR-mediated currents must activate much more quickly than reported rise times of $\sim 23-27 \mathrm{~ms}$ for NMDAR excitatory postsynaptic potentials in the rat IC (Ma et al., 2002; Wu et al., 2004). Elsewhere, in vitro and in vivo experiments support the presence of fast-acting NMDAR currents (D'Angelo et al., 1990; Wolszon et al., 1997). The fast action may depend on the location of NMDARs relative to the spike trigger zone of a neuron (D'Angelo et al., 1990). In IC neurons, dendritic placement of NMDARs may contribute to latency differences among long-latency neurons.

Based on the diverse effects of NMDAR blockade and on the above conclusions, we hypothesize that several structural and functional varieties of NMDARs must occur in IC. NMDARs with NR2A or NR2B subunits typically display a fast time course and high $\mathrm{Mg}^{2+}$ sensitivity (Mori and Mishina, 1995; Kirson et al., 1999). These subunit properties are consistent with IC responses in which short-latency spikes depend on both AMPARs and NMDARs. In contrast, NMDARs with NR2D subunits are associated with a slower time course and weaker voltage-dependant $\mathrm{Mg}^{2+}$ block (Monyer et al., 1994; Momiyama et al., 1996). These may be associated with IC neurons that show AMPARindependent NMDAR activity, especially neurons with long firstspike latencies. NR2D subunits are commonly associated with developing rather than adult brains (Kirson and Yaari, 1996), but significant levels occur in adult IC (Wenzel et al., 1996). Among IC neurons, the diversity of NMDA-mediated responses in time course and AMPA dependence suggests additional functional subtypes. Further study should examine both subunit expression by IC neurons as well as localization on postsynaptic neurons. 

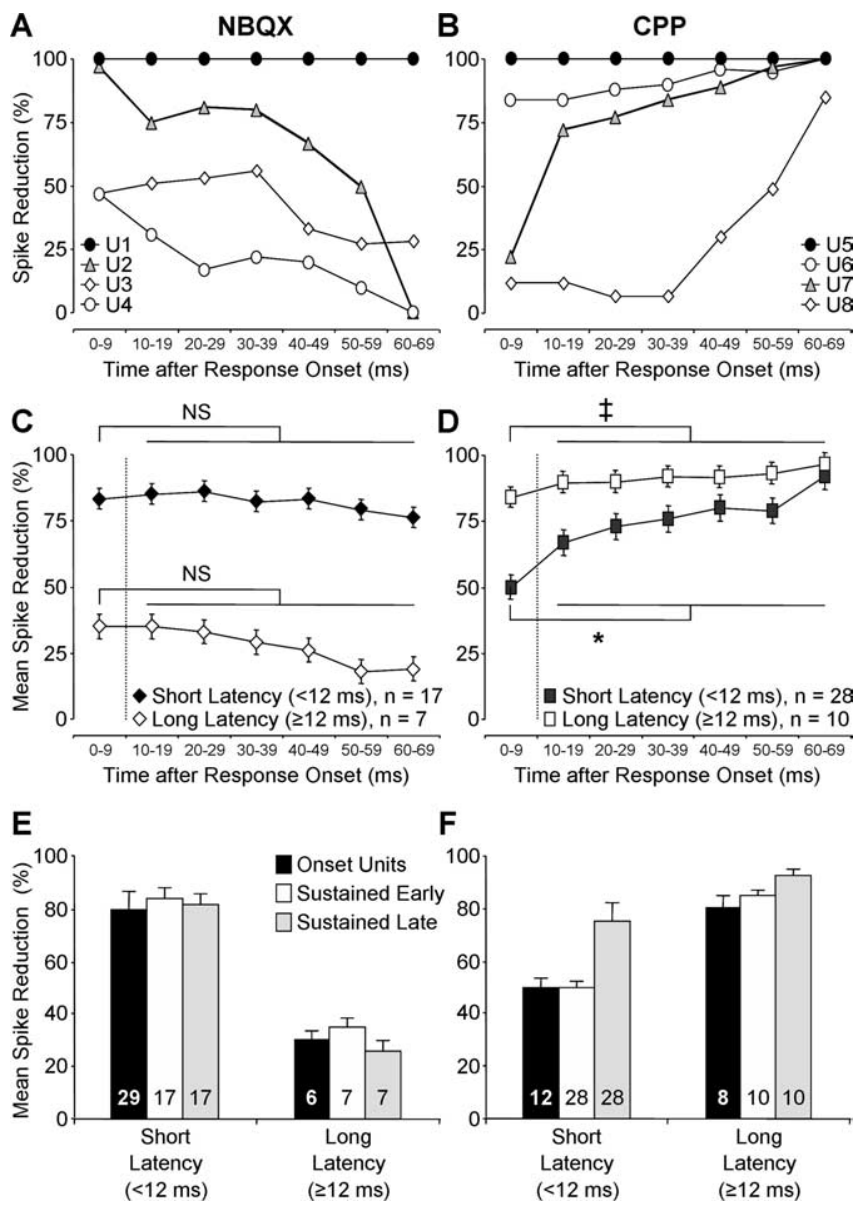

\section{$F$}

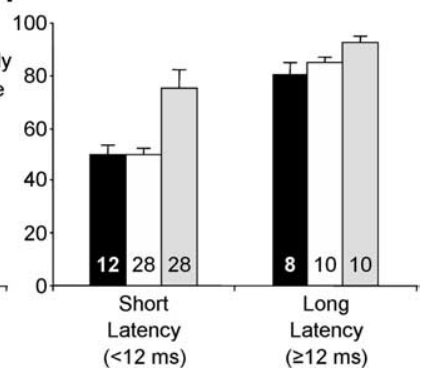

Figure 9. AMPAR and NMDAR blockade reduced spikes throughout the time course of responses in IC units. All plots display a reduction in auditory-evoked spikes resulting from drug application, analyzed in $10 \mathrm{~ms}$ blocks after initial response. $\boldsymbol{A}$, Effect of NBQX on auditoryevoked spikes in four sustained units. Variation in spike reduction by NBQX was partly related to differences in first-spike latency. Minimum latencies are as follows: U1, $7.7 \mathrm{~ms} ; \mathrm{U2}, 9.6 \mathrm{~ms} ; \mathrm{U3}$, $12.7 \mathrm{~ms} ; \mathrm{U} 4,14.8 \mathrm{~ms}$. $\boldsymbol{B}$, Effect of CPP on auditory-evoked spikes in four sustained units. Stronger CPP effects were observed in long-latency units. Minimum latencies are as follows: U5, 19.1 $\mathrm{ms}$; U6, $10.1 \mathrm{~ms} ; \mathrm{U7}, 9.2 \mathrm{~ms}$; U8, 7.8 ms. C, D, Average reductions in auditory-evoked spikes for the samples of short- and long-latency units. Drug differences were assessed using two-sample $t$ tests assuming unequal variances: NS, not significant; ${ }^{\ddagger} p<0.05 ;{ }^{*} p<0.01$. C, NBQX strongly reduced spikes throughout the duration of sustained responses in short-latency units but not in long-latency units. For each latency group, there was no difference in spike reduction for earlier and later components of the response. $D$, CPP strongly reduced spikes throughout the duration of sustained responses in both short- and long-latency samples but was more effective in long-latency units. Spike reduction was significantly greater for later spikes than for early spikes in both short-latency and long-latency samples. $\boldsymbol{E}$, For each latency group, spike reduction by NBQX was similar for onset units, early sustained spikes, and late sustained spikes. $\boldsymbol{F}$, For each latency group, spike reduction by CPP for onset units and the early component of sustained responses were similar.

\section{Roles of AMPARs and NMDARs in temporal patterns of response to sound}

As in other systems (Sillito et al., 1990), previous work in IC has emphasized a strong linkage between onset components of sensory-evoked responses and AMPAR-mediated currents (Faingold et al., 1989; Feldman and Knudsen, 1994; Zhang and Kelly, 2001; Ma et al., 2002). Our results show that this linkage is weak in the mustached bat's IC: onset responses can be AMPAR mediated, NMDAR mediated, or both. There are two reasons for this weak connection. First, some NMDARs in IC act more quickly than generally thought. Second, the substantial difference in first-spike latency across IC neurons means that onset re- sponses may occur early (within 5-6 ms of the input latency) or late (up to $40-50 \mathrm{~ms}$ after the input latency). Our results show that AMPARs contribute substantially to onset components of neural discharges, but only when these begin within 5-6 ms of the estimated input latency of $4-6 \mathrm{~ms}$. The results are thus consistent with the generally understood rapidity of AMPAR depolarization but establish the point that particular glutamate receptor currents are poorly related to the different temporal components of the response to sound of a neuron.

Sustained components of IC responses likewise have no exclusive association with NMDARs in neurons with first-spike latencies $<12 \mathrm{~ms}$. In these, AMPAR blockade eliminates spikes in the later, sustained component of temporal response patterns as effectively as it eliminates onset spikes. AMPARs may contribute to sustained spikes by providing an initial depolarization that removes $\mathrm{Mg}^{2+}$ block from NMDAR channels, as is generally understood. In these neurons, there may be no additional role of AMPARs, either because the glutamatergic input has an onset pattern or because AMPAR-mediated currents in response to later input spikes are reduced by synaptic depression (Brenowitz and Trussell, 2001; Wong et al., 2003). However, in some neurons, later spikes occur during blockade of NMDARs. Here, AMPARs may contribute directly to rapid depolarization that generates each later spike. For this to occur, the input spike pattern should be sustained, and the AMPAR excitation should be less susceptible to synaptic depression. Among different shortlatency neurons, AMPARs may contribute to later spikes through one or both of these mechanisms.

In contrast to sustained components of short-latency responses, sustained elements of long-latency responses show virtually no influence of AMPARs. As an example, for a neuron having a first-spike latency of $8 \mathrm{~ms}$ and sustained pattern, spikes occurring $50 \mathrm{~ms}$ after stimulus onset are highly sensitive to NBQX (Fig. $8 A$ ). In contrast, for a neuron with $15 \mathrm{~ms}$ first-spike latency and sustained discharge, spikes occurring $50 \mathrm{~ms}$ after stimulus onset are insensitive to NBQX (Fig. $8 B$ ). The difference is so stark that we believe it represents a major difference in the relative abundance or placement of AMPARs and NMDARs on the postsynaptic membranes of short- and long-latency neurons.

We propose that differential abundance/placement of AMPARs and NMDARs creates a distribution of response timing within IC. Previous hypotheses that addressed the origin of long first-spike latencies were based mostly on fast inhibition (Parks and Pollak, 1993; Casseday and Covey, 1996; Saitoh and Suga, 1995). However, fast inhibition generally increases first-spike latency by a small amount, usually $<2$ ms (Le Beau et al., 1996; Lu et al., 1997; Fuzessery et al., 2003). Variations in input latency obviously contribute to first-spike latency, but variation in latency of ascending inputs to IC is limited (Haplea et al., 1994; Klug et al., 2000). Neither factor accounts for longer first-spike latencies, up to $50 \mathrm{~ms}$ in IC (Parks and Pollak, 1993). Corticocollicular inputs could have longer latencies, and although some evidence suggests that cortical input can drive IC spikes (Syka and Popelar, 1984), most work suggests that cortical influence is modulatory (Jen et al., 1998; Suga et al., 2002). Other modulators of IC responses may alter first-spike latency [e.g., serotonin (Hurley and Pollak, 2005)] but are unlikely to both generate spikes from auditory input and delay the excitation. The slower time course of independent NMDA-mediated excitation has the appropriate features to explain how responses with longer firstspike latencies originate.

The roles of NMDARs in sensory information relay and processing are multiple. Previous workers focused on functions re- 
lated to effectiveness in activating synaptic targets (Blitz and Regehr, 2003), temporal integration (Zhang and Kelly, 2001; Wu et al., 2004), and enhanced responses to coincident input (Binns, 1999). This study suggests that the NMDARs may be used to regulate first-spike latency, an essential component in the analysis of temporally distributed elements in sensory stimuli and a necessary feature in matching the timing of sensory-evoked neural activity to appropriate behavioral responses (Casseday and Covey, 1996).

\section{References}

Binns KE (1999) The synaptic pharmacology underlying sensory processing in the superior colliculus. Prog Neurobiol 59:129-159.

Blitz DM, Regehr WG (2003) Retinogeniculate synaptic properties controlling spike number and timing in relay neuron. J Neurophysiol 90:2438-2450.

Brenowitz S, Trussell LO (2001) Minimizing synaptic depression by control of release probability. J Neurosci 21:1857-1867.

Casseday JH, Covey E (1996) A neuroethological theory of the operation of the inferior colliculus. Brain Behav Evol 47:311-336.

D’Angelo E, Rossi P, Garthwaite J (1990) Dual-component NMDA receptor currents at a single central synapse. Nature 346:467-470.

Davies J, Watkins JC (1983) Role of excitatory amino acid in mono- and polysynaptic excitation in the cat spinal cord. Exp Brain Res 49:280-290.

Faingold CL, Hoffman WE, Caspary DM (1989) Effects of excitatory amino acids on acoustic responses of inferior colliculus. Hear Res 40:127-136.

Feldman DE, Knudsen EI (1994) NMDA and non-NMDA glutamate receptors in auditory transmission in the barn owl inferior colliculus. J Neurosci 14:5939-5958.

Fleidervish IA, Binshtok AM, Gutnick MJ (1998) Functionally distinct NMDA receptors mediate horizontal connectivity within layer 4 of mouse barrel cortex. Neuron 21:1055-1065.

Fuzessery ZM, Wenstrup JJ, Hall JC, Leroy S (2003) Inhibition has little effect on response latencies in the inferior colliculus. J Assoc Res Otolaryngol 4:60-73.

Haplea S, Covey E, Casseday JH (1994) Frequency tuning and response latency at three levels in the brainstem of the echolocating bat, Eptesicus fuscus. J Comp Physiol 174:671-682.

Havey DC, Caspary DM (1980) A simple technique for constructing "piggyback" multibarrel microelectrodes. Electroencephalogr Clin Neurophysiol 48:249-251.

Heggelund P, Hartveit E (1990) Neurotransmitter receptors mediating excitatory inputs to cells in the cat lateral geniculate nucleus. I. Lagged cells. J Neurophysiol 63:1347-1360.

Hurley LM, Pollak GD (2005) Serotonin shifts first-spike latencies of inferior colliculus neuron. J Neurosci 24:7876-7886.

Jen PH, Chen QC, Sun XD (1998) Corticofugal regulation of auditory sensitivity in the bat inferior colliculus. J Comp Physiol A Neuroethol Sens Neural Behav Physiol 183:683-697.

Kelly JB, Zhang H (2002) Contribution of AMPA and NMDA receptors to excitatory responses in the inferior colliculus. Hear Res 168:35-42.

Kirson ED, Yaari Y (1996) Synaptic NMDA receptors in developing mouse hippocampal neurones: functional properties and sensitivity to ifenprodil. J Physiol (Lond) 497:437-455.

Kirson ED, Yaari Y (2000) Unique properties of NMDA receptors enhances synaptic excitation of radiatum giant cells in rat hippocampus. J Neurosci 20:4844-4854

Kirson ED, Schirra C, Konnerth A, Yaari Y (1999) Early postnatal switch in the magnesium sensitivity of NMDA receptors in rat CA1 pyramidal cells. J Neurophysiol 521:99-111.

Klug A, Khan A, Burger RM, Bauer EE, Hurley LM, Yang L, Grothe B, Halvorsen MB, Park TJ (2000) Latency as a function of intensity in auditory neurons: influence of central processing. Hear Res 148:107-123.

Kwon YH, Esguerra M, Mriganka S (1991) NMDA and non-NMDA recep- tors mediate visual responses of neurons in the cat's lateral geniculate nucleus. J Neurophysiol 66:414-428.

Langner G, Schreiner C, Merzenich MM (1987) Covariance of latency and temporal resolution in the inferior colliculus of the cat. Hear Res 31:197-202.

Le Beau FEN, Rees A, Malmierca MS (1996) Contribution of GABA- and glycine-mediated inhibition to the monaural temporal response properties of neuron in the inferior colliculus. J Neurophysiol 75:902-919.

Lodge D, Davies SN, Jones MG, Millar J, Manallack DT, Ornstein PL, Verberne AJ, Young N, Beart PM (1988) A comparison between the in vivo and in vitro activity of five potent and competitive NMDA antagonists. Br J Pharmacol 95:957-965.

Lu Y, Jen PH-S, Zhen Q-Y (1997) GABAergic disinhibition changes the recovery cycle of bat inferior colliculus neurons. J Comp Physiol 181:331-341.

Ma CL, Kelly JB, Wu SH (2002) AMPA and NMDA receptors mediate synaptic excitation in the rat's inferior colliculus. Hear Res 168:25-34.

Momiyama A, Feldmeyer D, Cull-Candy SG (1996) Identification of a native low-conductance NMDA channel with reduced sensitivity to $\mathrm{Mg}^{2+}$ in rat central neurons J Physiol (Lond) 494:479-492.

Monyer H, Burnashev N, Laurie DJ, Sakmann B, Seeburg PH (1994) Developmental and regional expression in the rat brain and functional properties of four NMDA receptors. Neuron 12:529-540.

Mori H, Mishina M (1995) Structure and function of the NMDA receptor channel. Neuropharmacology 34:1219-1237.

Nataraj K, Wenstrup JJ (2005) Roles of inhibition in creating complex auditory responses in the inferior colliculus: facilitated combinationsensitive neurons. J Neurophysiol 93:3294-3312.

Nataraj K, Wenstrup JJ (2006) Roles of inhibition in creating complex auditory responses in the inferior colliculus: inhibited combinationsensitive neurons. J Neurophysiol 95:2179-2192.

Ozawa S, Kamiya H, Tsuzuki K (1998) Glutamate receptors in the mammalian central nervous system. Prog Neurobiol 54:581-618.

Parks TJ, Pollak GD (1993) GABA shapes sensitivity to interaural intensity disparities in the mustache bat's inferior colliculus: implications of encoding sound location. J Neurosci 13:2050-2067.

Randle JC, Guet T, Cordi A, Lepagnol JM (1992) Competitive inhibition by NBQX of kainate/AMPA receptor currents and excitatory synaptic potentials: importance of 6-nitro substitution. Eur J Pharmacol 215:237-244.

Saitoh I, Suga N (1995) Long delay lines for ranging are created by inhibition in the inferior colliculus of the mustached bat. J Neurophysiol 74:1-11.

Sillito AM, Murphy PC, Salt TE, Moody CI (1990) Dependence of retinogeniculate transmission in cat on NMDA receptors. J Neurophysiol 63:347-355.

Sivaramakrishnan S, Oliver DL (2005) Neural responses to lemniscal stimulation in laminar brain slices of the inferior colliculus. J Assoc Res Otolaryngol 7:1-14.

Suga N, Xiao Z, Ma X, Ji W (2002) Plasticity and corticofugal modulation for hearing in adult animals. Neuron 36:9-18.

Syka J, Popelar J (1984) Inferior colliculus in the rat: neuronal responses to stimulation of the auditory cortex. Neurosci Lett 15:235-240.

Wenzel A, Villa H, Mohler D, Benke D (1996) Developmental and regional expression of NMDA receptor subtype containing the NR2D subunit in rat brain. J Neurochem 66:1240-1248.

Wolszon LR, Pereda AE, Faber DS (1997) A fast synaptic potential mediated by NMDA and non-NMDA receptors. J Neurophysiol 78:2693-2706.

Wong AY, Graham BP, Billups B, Forsythe ID (2003) Distinguishing between presynaptic and postsynaptic mechanisms of short-term depression during action potential trains. J Neurosci 23:4868-4877.

Wu SH, Ma CL, Kelly JB (2004) Contribution of AMPA, NMDA, and $\mathrm{GABA}_{\mathrm{A}}$ receptors to temporal pattern of postsynaptic responses in the inferior colliculus of the rat. J Neurosci 24:4625-4634.

Zhang H, Kelly JB (2001) AMPA and NMDA receptors regulate responses of neurons in the rat's inferior colliculus. J Neurophysiol 86:871-880. 